\title{
IrRECIST Stable Disease
}

National Cancer Institute

\section{Source}

National Cancer Institute. irRECIST Stable Disease. NCI Thesaurus. Code C140317.

A failure to meet criteria for irCR or irPR in the absence of irPD. 